\title{
Mitigating artifacts in back-projection source imaging with implications for frequency-dependent properties of the Tohoku-Oki earthquake
}

\author{
Lingsen Meng ${ }^{1}$, Jean-Paul Ampuero ${ }^{1}$, Yingdi Luo ${ }^{1}$, Wenbo $\mathrm{Wu}^{2}$, and Sidao $\mathrm{Ni}^{3}$ \\ ${ }^{1}$ Seismological Laboratory, California Institute of Technology, Pasadena, California 91125, U.S.A. \\ ${ }^{2}$ Department of Earth and Space Sciences, University of Science and Technology of China, Hefei, Anhui 230026, P.R. China \\ ${ }^{3}$ CAS Key Laboratory of Dynamical Geodesy, Institute of Geodesy and Geophysics, Wuhan 430077, China
}

(Received December 27, 2011; Revised May 4, 2012; Accepted May 17, 2012; Online published January 28, 2013)

\begin{abstract}
Comparing teleseismic array back-projection source images of the 2011 Tohoku-Oki earthquake with results from static and kinematic finite source inversions has revealed little overlap between the regions of high- and low-frequency slip. Motivated by this interesting observation, back-projection studies extended to intermediate frequencies, down to about $0.1 \mathrm{~Hz}$, have suggested that a progressive transition of rupture properties as a function of frequency is observable. Here, by adapting the concept of array response function to non-stationary signals, we demonstrate that the "swimming artifact", a systematic drift resulting from signal non-stationarity, induces significant bias on beamforming back-projection at low frequencies. We introduce a "reference window strategy" into the multitaper-MUSIC back-projection technique and significantly mitigate the "swimming artifact" at high frequencies ( $1 \mathrm{~s}$ to $4 \mathrm{~s}$ ). At lower frequencies, this modification yields notable, but significantly smaller, artifacts than time-domain stacking. We perform extensive synthetic tests that include a 3D regional velocity model for Japan. We analyze the recordings of the Tohoku-Oki earthquake at the USArray and at the European array at periods from $1 \mathrm{~s}$ to $16 \mathrm{~s}$. The migration of the source location as a function of period, regardless of the backprojection methods, has characteristics that are consistent with the expected effect of the "swimming artifact". In particular, the apparent up-dip migration as a function of frequency obtained with the USArray can be explained by the "swimming artifact". This indicates that the most substantial frequency-dependence of the Tohoku-Oki earthquake source occurs at periods longer than $16 \mathrm{~s}$. Thus, low-frequency back-projection needs to be further tested and validated in order to contribute to the characterization of frequency-dependent rupture properties.
\end{abstract}

Key words: Back-projection, Tohoku-Oki earthquake, frequency-dependent rupture properties, MUSIC, swimming artifact.

\section{Introduction}

As one of the most important earthquakes in the history of seismology, the 2011 M9 Tohoku-Oki earthquake enables a broad spectrum of studies of the physics of devastating subduction earthquake giants. One key feature observed in this earthquake is that most of the low-frequency (LF) slip is located up-dip from the hypocenter. This is supported by teleseismic and geodetic source inversions (Koketsu et al., 2011; Lee et al., 2011; Shao et al., 2011; Simons et al., 2011; Yue and Lay, 2011), seafloor displacement measurement (Sato et al., 2011) and Rayleigh wave back-projection (Roten et al., 2011). In contrast, the high frequency (HF) slip is distributed at the bottom of the seismogenic zone. This is inferred from teleseismic back-projection (Ishii, 2011; Koper et al., 2011b; Meng et al., 2011; Wang and Mori, 2011; Yao et al., 2011) and local strong motion studies (Asano and Iwata, 2011; Meng et al., 2011; Miyake et al., 2011). This frequency-dependent rupture behavior is reported by (Nakahara et al., 2008) for a number of earthquakes and has also been proposed for the 2004 Suma-

Copyright (c) The Society of Geomagnetism and Earth, Planetary and Space Sciences (SGEPSS); The Seismological Society of Japan; The Volcanological Society of Japan; The Geodetic Society of Japan; The Japanese Society for Planetary Sciences; TERRAPUB.

doi:10.5047/eps.2012.05.010 tra and 2010 Maule earthquakes (Lay et al., 2012). This spatial complementarity between low- and high-frequency slip provides interesting constraints for physical models of earthquake rupture (Kato, 2007; Huang et al., 2012) and is important for strong motion prediction. Meng et al. (2011) related this observation to rheological heterogeneities in a broad brittle ductile transition zone.

Motivated by the spatial complementarity between LF slip $(<0.1 \mathrm{~Hz})$ and HF slip $(\sim 1 \mathrm{~Hz})$, a number of studies (Koper et al., 2011a; Mori, 2011; Yao et al., 2011; Lay et $a l ., 2012)$ attempted to probe the transition between these two frequency ranges by performing back-projection at intermediate frequencies. Their results based on the USArray recordings suggest a progressive up-dip migration of the dominant sources as a function of decreasing frequency from 1 to $0.1 \mathrm{~Hz}$. As plausible as this finding is, the backprojection at low frequencies around $0.1 \mathrm{~Hz}$ needs to be validated. A potential source of bias addressed here is the so-called "swimming artifact" (Ishii et al., 2007; Walker and Shearer, 2009; Xu et al., 2009; Yao et al., 2012) which appears as a frequency-dependent migration artifact due to the non-stationarity of seismic signals. This artifact is a well-known problem in the back-projection community. It degrades the quality of the images and makes it difficult to build confidence on the fine details of the source imag- 
ing, especially when the features of interest have a similar migration direction as the artifact. A common practice is to smooth the images to suppress the artifact (Koper et al., 2011a), but this is not fully successful and comes at the expense of resolution.

In this study, by introducing a modified form of the array response function for non-stationary signals and synthetic tests that incorporate regional velocity models for Japan, we show that the "swimming artifact" is prominent in low-frequency beamforming back-projection and can lead to apparent frequency-dependent rupture behavior. On the other hand, by adopting a "reference window strategy" in the frequency-domain MUSIC back-projection (Meng et al., 2011), we are able to compensate the signal non-stationarity and mitigate the swimming artifacts at high frequency $(\sim 1 \mathrm{~Hz})$ and significantly reduce them at relative low frequencies $(\sim 0.1 \mathrm{~Hz})$. Based on extensive synthetic tests and improved results from the USArray and European array, we find that the frequency-dependent source offset observed for the Tohoku-Oki earthquake is consistent with the expected effect of the artifact regardless of the backprojection methods. We thus conclude with a cautionary note on the inference of frequency-dependent source properties from low-frequency back-projection studies.

\section{2. "Swimming" Effect in Back-Projection of Non- Stationary Signals}

\subsection{Non-stationary array response function}

The basic tool to assess the resolution capability of a seismic array is the array response function (ARF) defined as the linear beamforming amplitude as a function of source location (or direction of arrival theta) offset with respect to the true location of a point source, under the assumption of a stationary signal with perfect coherence across the array (e.g. Rost and Thomas, 2002):

$$
A(\theta)=\left|\sum_{k} e^{i \omega t_{k}(\theta)}\right|^{2}
$$

where $\omega$ is the angular frequency, $t_{k}$ is the the time delay at the $k$ th station as a function of relative location $\theta$ of the trial source. In principle, the true source location brings in phase the signal at all the stations and yields the maximum array beamforming output. The ARF of 2D arrays typically shows an elliptical main lobe, whose size is proportional to the wavelength-to-aperture ratio and defines the resolution limit of standard beamforming. In reality, earthquake waveforms are far from being stationary. Their envelope amplitude decays as a function of time due to scattering in the heterogeneous crust (Sato and Fehler, 1998; Zerva and Zervas, 2002). To account for the non-stationarity of the seismic signal, we propose a modified ARF by introducing an additional time dimension and a decaying weight function $S(t)$ that represents the typical decay of the waveform envelopes:

$$
A(\theta)=\left|\sum_{k} S\left(t_{k}(\theta)\right) e^{i \omega t_{k}(\theta)}\right|^{2} .
$$

The function $S(t)$ can be rather complicated since it involves both the amplitude and phase perturbation. Further- more, in general this function differs from station to station due to site effects. To simplify the presentation, we consider that $S(t)$ is the same for all the stations in the array and adopt a decaying exponential function. We estimate its characteristic decay time as a function of frequency by fitting a decaying exponential to the envelope of the stacked USArray seismograms of a $M_{\mathrm{w}} 7.1$ foreshock (on March 9, 2011) in different frequency bands. Introducing this decay function into the ARF represents the beamforming of a signal with an exponentially decaying envelope starting at $t=0$ (Fig. 1(c)).

\subsection{The origin of the "swimming artifact"}

We study the modified ARF in a 2D Earth configuration first. A linear array comprising of 16 stations is located at teleseismic distances, from $75^{\circ}$ to $90^{\circ}$ away from the hypocenter of a point source. The stations are regularly spaced by $1^{\circ}$ (Fig. 1(a)). We computed the modified ARF in different frequency bands. Figure 1(b) shows that the main lobe of the modified ARF migrates as a function of time towards the direction of the array. This is the hallmark of an artifact that has been referred to in previous backprojection studies as the "swimming artifact" (Ishii et al., 2007; Walker and Shearer, 2009; Xu et al., 2009; Yao et al., 2012). Our analysis shows that the artifact is caused by the non-stationarity of the signals.

Although the decay rate of $S(t)$ controls the severity of the artifact, the existence of the bias is inevitable as long as the signal power decays as a function of time. In particular, its existence does not depend on the detailed shape of the decay function. The origin of the artifact can be understood as follows. We consider the true source location $\mathrm{A}$ and a trial location B closer to the array. At $t=0 \mathrm{~s}$, when the signal envelope reaches its maximum, the travel time curve from source A samples the maximum of the signal envelope at all stations and leads to a maximum stack. Thus, the peak of the beam gives a correct estimate of the true location. Later on, for instance at $t=10 \mathrm{~s}$, the travel time curve from A still aligns the array signals with a uniform, although lower, amplitude. However, the travel time curve from source B (dashed line in Fig. 1(a)) samples also the earlier, larger amplitude part of the waveform. Although it renders the stations slightly out of phase, it gives a larger stack than a trial source on A at $t=10$ (solid line in Fig. 1(b)). Thus, the modified ARF at B is larger than at A, which creates an apparent shift in the estimated source location. The speed of the drift scales with wavelength, so it is amplified at a lower frequency. For a given travel time curve, the phase perturbation is smaller at a longer period, resulting in the peak sum appearing even further off the true location. Although our analysis is based on the array response, a concept belonging to standard beamforming, this phenomenon is common to all array processing methods, since they are all based on the signal coherency and amplitude.

The "swimming artifact" in this 2D Earth example leads to the naive interpretation that the bias occurs in the sourcearray direction, which is in the along-strike direction when imaging the Tohoku-Oki earthquake with the USArray. However, in the 3D Earth case, the direction and strength of the artifact are controlled by both the source-to-array di- 


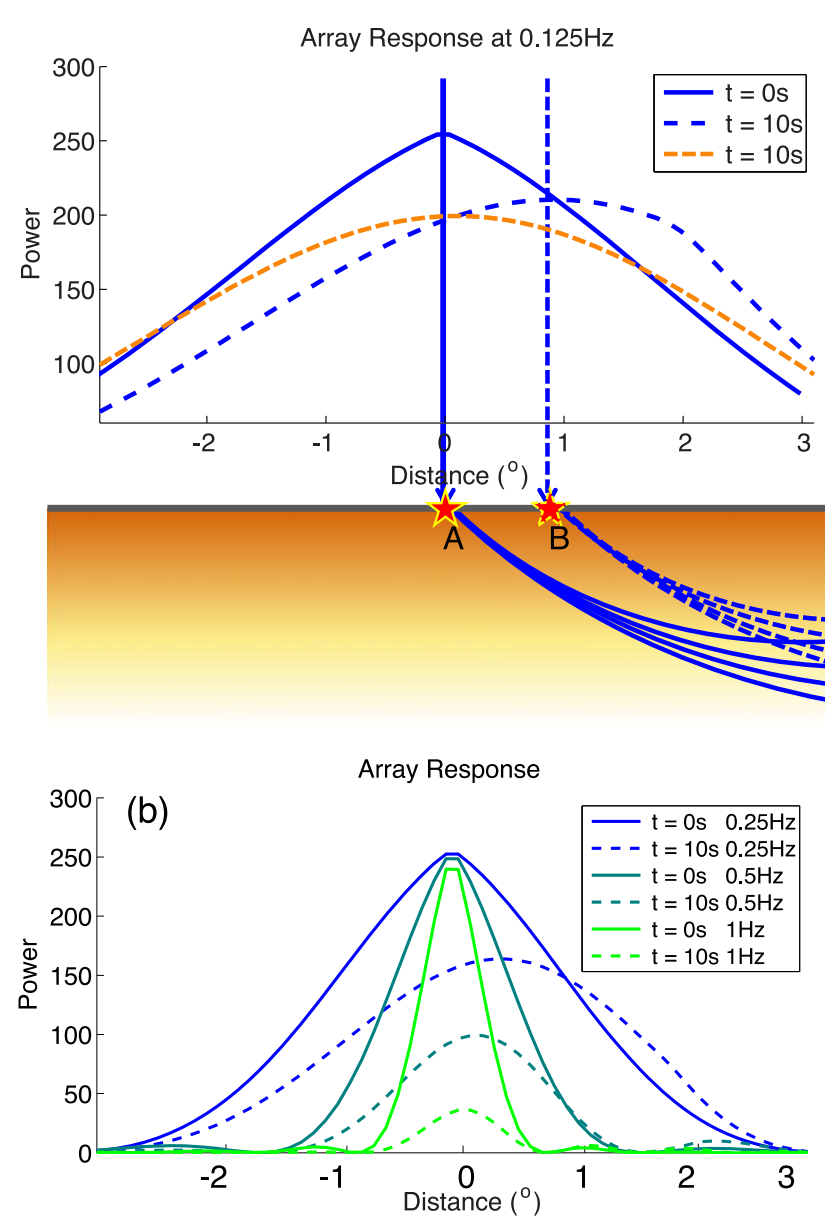

(a)

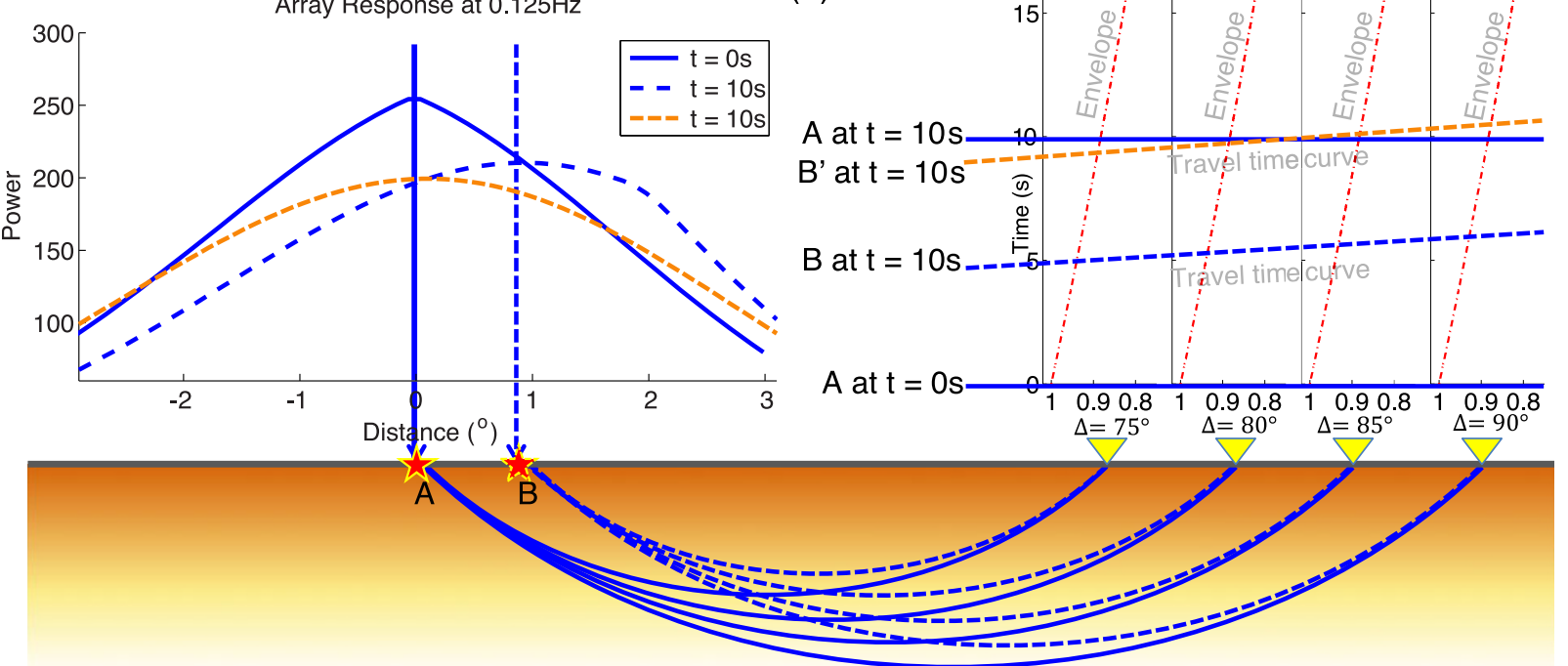

Exponential Fit

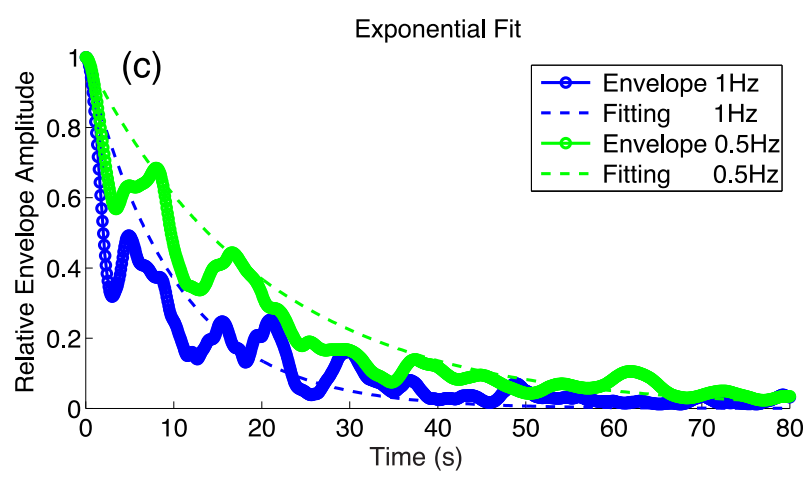

Fig. 1. The 'swimming' artifact of a 1D array in a 2D Earth. (a) 'Swimming' effect at a lower frequency $(0.125 \mathrm{~Hz})$ with respect to a teleseismic $\left(75^{\circ}-90^{\circ}\right)$ linear array of 16 stations $\left(1^{\circ}\right.$ spacing). Upper left figure shows the artifact: at $t=10 \mathrm{~s}$, maximum array response drifted from the true location $\mathrm{A}\left(0^{\circ}\right.$, as solid-edged star) to the apparent location B $\left(0.9^{\circ}\right.$, dash-edged star), a difference of about $100 \mathrm{~km}$. Upper right figure shows the traveling time curve sampling envelopes of stations \#1, 6, 11 and 16 (distance $75^{\circ}, 80^{\circ}, 85^{\circ}, 90^{\circ}$, respectively, plotted as yellow triangles). The solid and dashed abscissas denote the travel time curve of a hypothetical source occurring at a certain location and origin time. At $t=10 \mathrm{~s}$, the blue dashed line is the traveling time curve that yields the overall maximum of the array response, taking into account the signal decay. Instead of the true source location A, it introduces an apparent location B closer to the array. On the other hand, the array response of the travel of B' (reference window strategy) is smaller than that of A, therefore no swimming artifact is created. (b) 'Swimming' effect in a different frequency band, with solid curves showing $t=0 \mathrm{~s}$, and dashed curves showing $t=10 \mathrm{~s}$, respectively. Note that, at all the frequencies, the maximum of array response shifts towards the array direction, this effect is reduced at a higher frequency. (c) Exponential-fitting estimation of the frequency-dependent time-decay function. Envelopes of the stacked signals are smoothed with a time window 2.5 times the upper period bound of the bandpass filter. Each envelope is aligned to $t=0$ and normalized with respect to its maximum. Due to the contamination of the signals by the micro-seisms, only signals higher than $0.25 \mathrm{~Hz}$ are analyzed. An attenuation decay function $e^{-0.1 f t}$ is estimated and used for all frequencies in this paper.

rection and the array configuration. Figure 2 shows that the artifact is most prominent along the longer axis of the array response for both the USArray and European array. The configuration of the two arrays can be found in Fig. 3. The ARF of the USArray extends along the E-W direction due to its dominantly longitudinal station distribution. Hence, the swimming artifact operates along dip (Fig. 2). This can potentially perturb any attempt to image frequency dependent along-dip location of slip.

\subsection{Mitigating the artifact}

Based on our developed understanding of the origin of the "swimming artifact", we can now propose a mitigation technique. Since the artifact is due to the fact that the windows corresponding to different trial slownesses sample different amplitudes of the non-stationary signal envelope, we propose a certain choice of windowing to minimize the effect of the signal non-stationarity. We call this technique a "reference window" approach.

The idea is to sample the recordings with the window corresponding to a reference slowness, usually corresponding to the hypocenter. When testing different slownesses (corresponding to different trial source locations) instead of using the actual window defined by the trial differential arrival times, we phase shift the data inside the reference window in the Fourier domain. Because this approach tests different slownesses with the same segment of the waveforms, the result is not affected by the temporal non-stationarity of the signals and, therefore, the swimming artifact is significantly reduced. Our reference window approach can be understood in the example in Fig. 1. At $t=10 \mathrm{~s}$, when testing the travel time curve corresponding to location B, instead of using the actual curve (blue dashed line in Fig. 1(a)) we test a curve $B^{\prime}$ (orange dashed line in Fig. 1(a)) that has the same slope as B but whose mean is adjusted to that of curve 


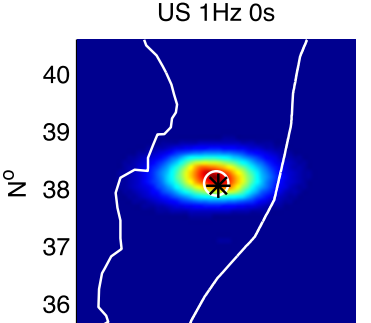

US $0.5 \mathrm{~Hz}$ Os

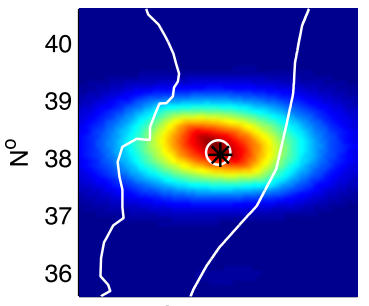

US $0.25 \mathrm{~Hz} 0 \mathrm{~s}$

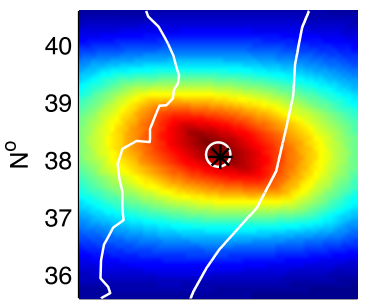

US $0.125 \mathrm{~Hz}$ 0s

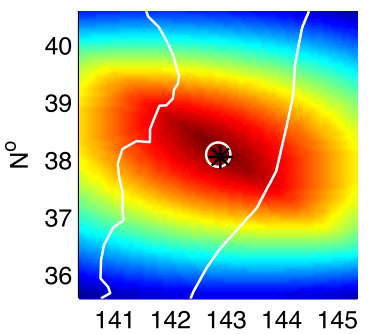

$\mathrm{E}^{\mathrm{O}}$
US $1 \mathrm{~Hz} 20 \mathrm{~s}$

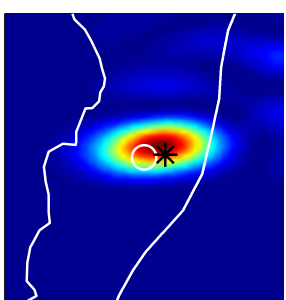

US $0.5 \mathrm{~Hz} 20 \mathrm{~s}$

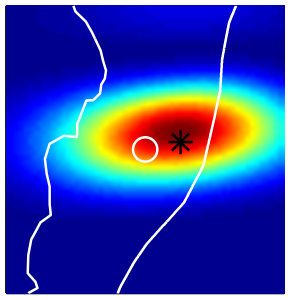

US $0.25 \mathrm{~Hz} 20 \mathrm{~s}$

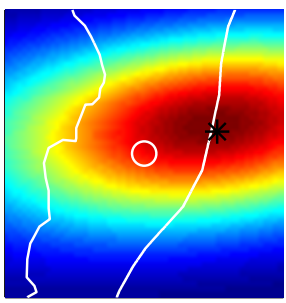

US $0.125 \mathrm{~Hz} 20 \mathrm{~s}$

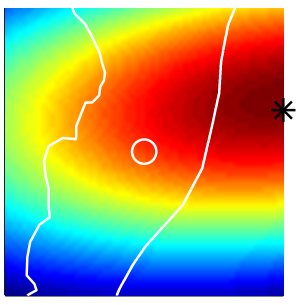

$\begin{array}{lllll}141 & 142 & 143 & 144 & 145\end{array}$ $E^{\circ}$
EU $1 \mathrm{~Hz}$ 0s

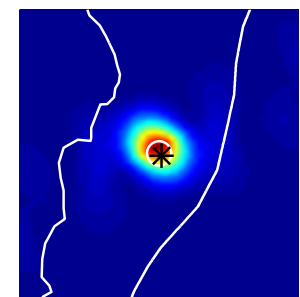

EU $0.5 \mathrm{~Hz}$ 0s

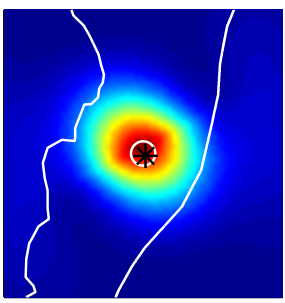

EU $0.25 \mathrm{~Hz}$ 0s

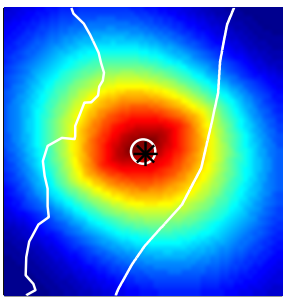

EU $0.125 \mathrm{~Hz}$ 0s

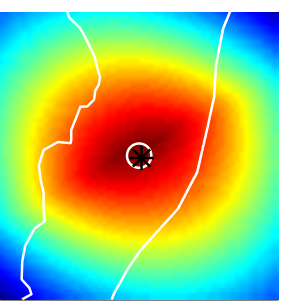

$141 \quad 142 \quad 143 \quad 144$

$E^{\circ}$
EU 1Hz 20s

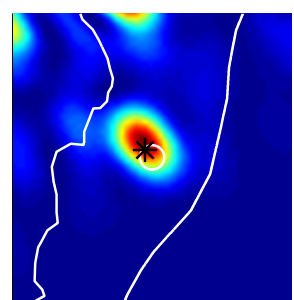

EU $0.5 \mathrm{~Hz} 20 \mathrm{~s}$

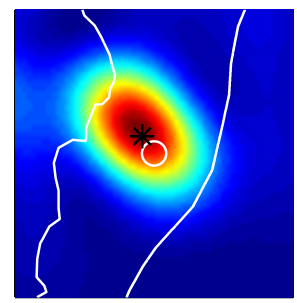

EU $0.25 \mathrm{~Hz} 20 \mathrm{~s}$

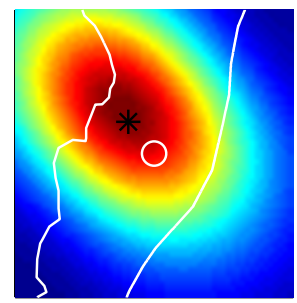

EU $0.125 \mathrm{~Hz} 20 \mathrm{~s}$

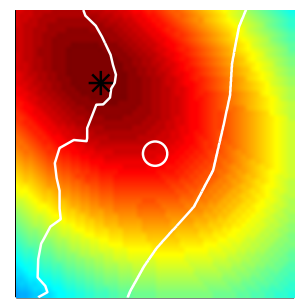

$141 \quad 142 \quad 143 \quad 144$

$E^{\circ}$

Fig. 2. Swimming effect in the 2D array response of the USArray (left two columns) and European array (right two columns) at frequencies of 1 , 0.5, 0.25 and $0.125 \mathrm{~Hz}$, with a color-coded array response normalized by its maximum, the white circle indicates the epicenter and a black star indicates the location of the maximum array response. Note that the swimming effect intensifies as the frequency decreases as in the 1D case, but the maximum swims in a direction that combines the longer axis of the array response and the source-to-array direction.

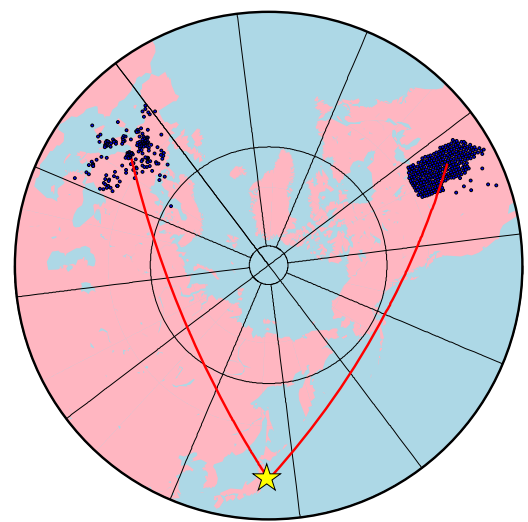

Fig. 3. Selected stations of the USArray and European array are denoted as the blue dots. The JMA hypocenter of the Tohoku-Oki is the yellow asterisk. The red lines are the great circle path from the centroid of each array to the hypocenter. The two arrays are both within $75^{\circ} \sim 90^{\circ}$ from the hypocenter.
A. This curve $\mathrm{B}^{\prime}$ corresponds to the same location as B but has a delayed absolute source origin time. Curves A and $\mathrm{B}^{\prime}$ sample the same part of the waveform and thus the same amplitude of the signal. Curve $\mathrm{B}^{\prime}$ has a smaller stack than curve A since it is off phase. Therefore the correct location $\mathrm{A}$ is recovered and no artifact is introduced.

The reference window strategy is embedded in frequency-domain back-projection techniques, such as the frequency-domain beamforming, MUSIC (Meng et al., 2011) and compressive sensing (Yao et al., 2011), in which the Fourier coefficients, or the covariance matrix, are computed based on the signals in the reference window. Because the Fourier shift implies periodic signals, the phase shift should not be too long relative to the window. Hence, the method is appropriate provided that the differential arrival times across the array are small compared to the length of the window. This is true when the sizes of the array and the source region are small compared to the 

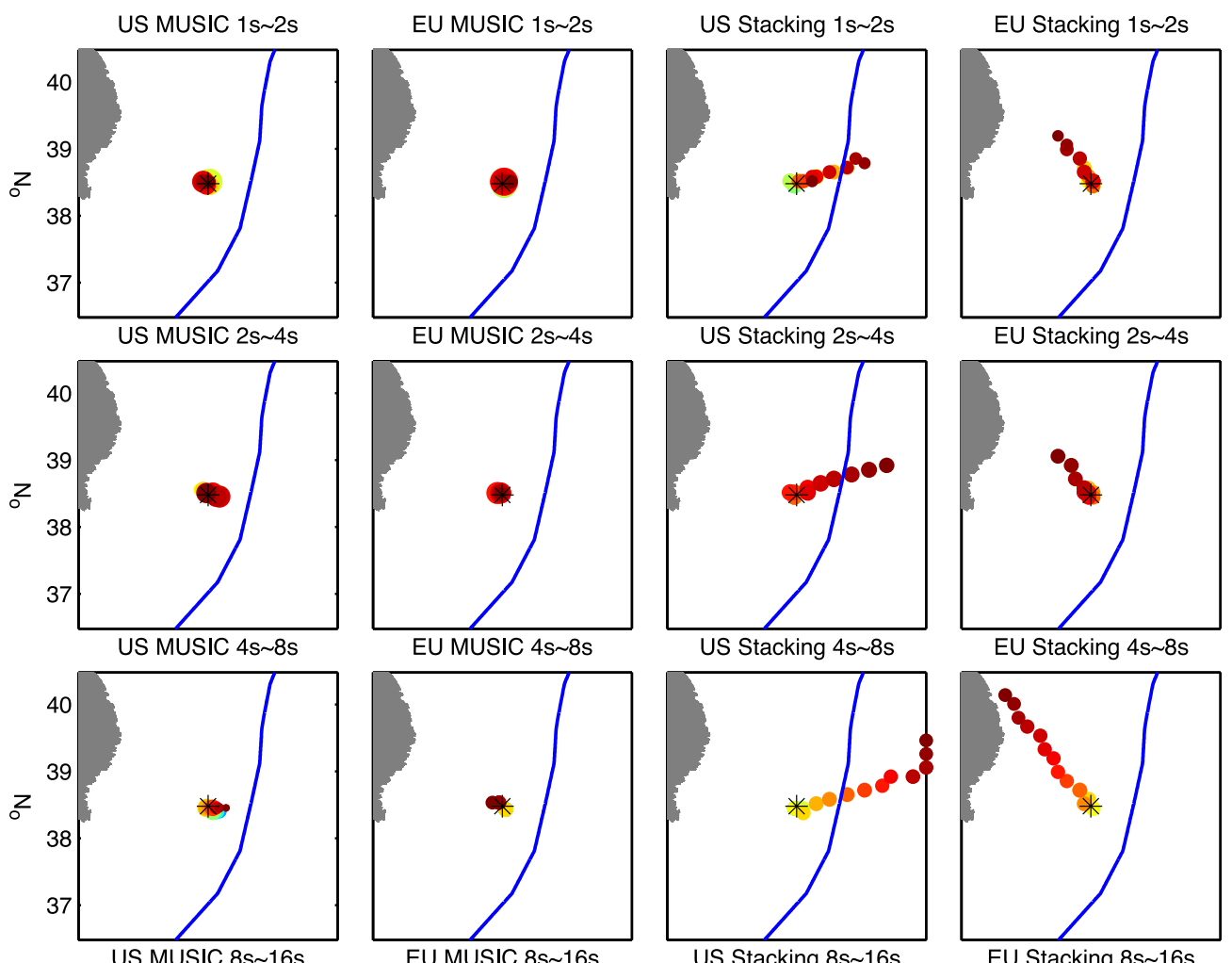

US Stacking $4 \mathrm{~s} \sim 8 \mathrm{~s}$

EU Stacking $4 \mathrm{~s} \sim 8 \mathrm{~s}$
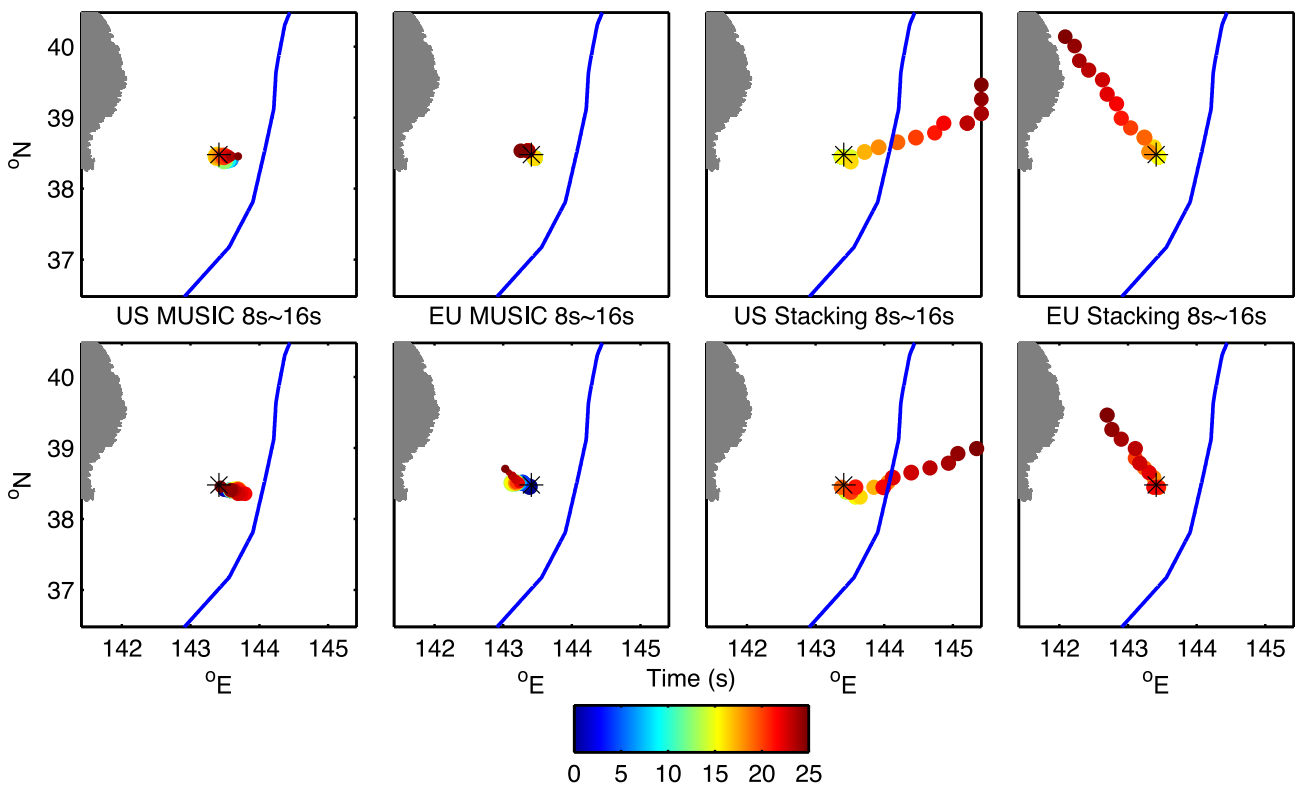

EU Stacking $8 s \sim 16 s$

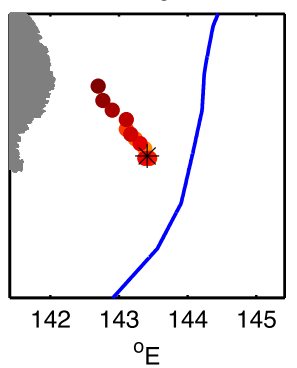

Fig. 4. Back-projection of aftershock synthetics. The synthetic aftershock seismograms at the USArray (left two columns) and European array (right two columns) are processed by the MUSIC and linear stacking techniques separately at periods of $1 \sim 2 \mathrm{~s}, 2 \sim 4 \mathrm{~s}, 4 \sim 8 \mathrm{~s}$ and $8 \sim 16 \mathrm{~s}$. The location of the aftershock is denoted by the asterisk. The solid circles are the peak of the back-projection images color-coded by time and sized by their relative amplitude with respect to the global maximum. The beamforming results show a "swimming" artifact at all frequencies. The MUSIC estimates are reliable at periods shorter than $4 \mathrm{~s}$. At lower frequencies, the spatial bias is notable but less severe than that of beamforming.

distance that $P$-waves travel over the window duration. For the Tohoku-Oki earthquake imaged by the European array and USArray at teleseismic distance, if the trial source region is up to 2 degrees from the epicenter, the standard deviation of the travel time curve is smaller than $1 \mathrm{~s}$, which is a small fraction of the $10 \mathrm{~s}$ long window. Hence, the reference window approach is valid in this case.

The reference window approach introduces a time shift, the difference between the mean of $\mathrm{B}$ and $\mathrm{B}^{\prime}$, therefore a timing correction is needed (Yao et al., 2011). This correction is readily implemented once the radiator locations have been identified in each back-projection image frame. The timing shown in our summary maps of HF radiation incorporates this correction, in which the true source time (color coded) of each identified radiator equals the timing of the back-projection image frame minus the mean of the travel time curve corresponding to the location of the radi- ator. In the next section, we conduct a series of synthetic tests to compare the frequency domain MUSIC (reference window) and time domain stacking (absolute window).

\section{Synthetic Test of Back-Projection at Various Frequencies \\ 3.1 Point source synthetic test}

To demonstrate the artifact at various frequencies and how well it can be mitigated by MUSIC, we test the backprojection on the synthetic USArray and European array seismograms of a $M 6.2$ aftershock with hypocenter location at $143.41^{\circ} \mathrm{E}, 38.32^{\circ} \mathrm{N}$. The station distributions of the two arrays are shown in Fig. 3. The synthetics are computed by a hybrid approach in which the wavefield in the source region (a $1100 \mathrm{~km} \times 1000 \mathrm{~km} \times 70 \mathrm{~km}$ box) is computed by the spectral element method (SPECFEM3D, Tromp et al., 2008) and interfaced with the geometrical ray theory 
up to the teleseismic receivers (Helmberger, 1983). This approach enables incorporating the complexity of regional velocity structures at an affordable computational cost and is ideal for testing teleseismic back-projection techniques at relatively high frequencies. In the SPECFEM3D simulation we used a regional tomography model (Miura et al., 2005) to account for waveform complexity introduced by 3D structures. The synthetic seismograms are computed with a sampling rate of $5 \mathrm{~Hz}$, but, due to the limited computation power, accuracy in the SPECFEM simulation is warranted only up to $1 \mathrm{~Hz}$. Future work involves incorporating realistic small-scale heterogeneities in the velocity model under the receivers to model waveform incoherence and coda decay.

Figure 4 shows the results of MUSIC and time-domain stacking back-projection of the aftershock scenario at various frequency bands. As expected from the modified ARF, the peak of the beamforming power progressively migrates along the major axis of the array response. As in our idealized ARF analysis, the peak amplitude decreases as it swims. On the other hand, the spatial bias of the MUSIC analysis at high frequencies $(0.25 \sim 1 \mathrm{~Hz})$ is negligible. At frequencies lower than $0.25 \mathrm{~Hz}$, the location shift is notable but substantially smaller than in time-domain stacking. Hence, at low frequency the "swimming" artifact cannot be ignored even with the MUSIC approach.

\subsection{Kinematic source synthetic test}

To further understand the artifact under more complicated and realistic circumstances, we conduct a kinematic rupture scenario involving two branches of simultaneous rupture along a circular rim (Fig. 5). The circle is centered at the hypocenter of the $M 6.2$ aftershock with a radius of $100 \mathrm{~km}$. It is divided into two semicircles by a line trending $210^{\circ}$ through the center. Two simultaneous North-to-South ruptures develop along each semicircle with a rupture speed of $2 \mathrm{~km} / \mathrm{s}$. Physically, this scenario is inspired by dynamic simulations in which a rupture front surrounds a circular asperity, or low-stress region, before breaking it (Dunham et al., 2003). The ruptures are composed of moving sources with a spatial interval of $40 \mathrm{~km}$. This spacing is chosen so that the sources are dense enough to represent a continuous rupture yet the coda wave is still substantial to maintain realistic signal non-stationarity. The Green's functions are the same as for the $M 6.2$ aftershock. This circular rupture model is relatively simple but has enough complexity, including simultaneous sources with a temporally varying spacing.

Figure 5 shows the results of MUSIC and linear beamforming back-projection of the circular rupture scenario with seismograms computed for both USArray and European array at periods from $2 \mathrm{~s}$ to $16 \mathrm{~s}$. In order to compare with results by Yao et al. (2011), we set the time window to be $10 \mathrm{~s}$ at the band of $1-4 \mathrm{~s}$ and $20 \mathrm{~s}$ for $4-16 \mathrm{~s}$. The locations of the first and second strongest radiators detected by both arrays in each window are plotted and color-coded by time. The size of the radiators is normalized by the maximum of the beamforming power or MUSIC pseudospectrum over the first $150 \mathrm{~s}$ of the rupture. In the highest frequency band $(0.5-1 \mathrm{~Hz})$, the MUSIC result of the European array almost exactly reproduces the synthetic rupture scenario, recovering radiators on the edge of the circle. The USArray result is noisier and distributed within the circle because its Green's functions have a smaller coherent arrival to coda ratio resulting from the nodal orientation of the array with respect to the focal mechanism. In the period band of $2 \mathrm{~s}$ to $4 \mathrm{~s}$, the MUSIC result of both arrays are within the circle. In the low-frequency band ( $8 \mathrm{~s}-16 \mathrm{~s})$, the uncertainties become large due to limited resolution and the swimming bias starts to be notable.

In comparison, beamforming is less capable of resolving the simultaneous sources. The recovered radiators are distributed along the expected artifact direction. Here, we also applied a common post-processing technique to weaken the swimming artifact (Koper et al., 2011b). The idea is that although the artifact swims, the true location always has the largest beamformed power. Thus, plotting the high frequency power against time and picking only the local maximum can, in principle, discriminate the true source location from the spurious sources. The processing is useful when the source process is simple, for instance for a fixed point source or a unilateral rupture, but less effective in complicated rupture scenarios involving multiple simultaneous sources. In the case of the circular rupture scenario, at relatively short periods ( $1 \mathrm{~s} \sim 4 \mathrm{~s})$ the identified radiators are located inbetween the true locations. At longer periods (4s $\sim 16 \mathrm{~s}$ ) they show considerable bias "downstream" of the true locations along the artifact direction, creating an apparently frequency-dependent rupture pattern.

\section{Low-Frequency Back-Projection of the Tohoku-Oki Earthquake}

The along-dip frequency dependent slip migration observed in previous back-projection studies is based on the USArray data (Koper et al., 2011a; Mori, 2011; Yao et al., 2011). The other large regional array, the European array, has been processed at high frequency around $1 \mathrm{~Hz}$ (Koper et al., 2011b; Meng et al., 2011). Analysis of the European array data at longer periods has not been reported before. Here, we back-project the Tohoku-Oki earthquake data recorded by the European array and USArray at periods from $2 \mathrm{~s}$ to $16 \mathrm{~s}$ with both the MUSIC and linear beamforming methods (Fig. 6). In order to be comparable to Yao et al. (2011), we set the beamforming time window to be $10 \mathrm{~s}$ at the band of $1-4 \mathrm{~s}$, and $20 \mathrm{~s}$ for 4 $16 \mathrm{~s}$. The locations of the radiators detected by both arrays in each frequency band are shown in Fig. 6. The size of the radiators is normalized by the maximum of the beamforming power or MUSIC pseudo-spectrum over the first $150 \mathrm{~s}$ of the rupture. For time-domain stacking, similar to the point source synthetic test, we observe a strong swimming effect at periods longer than $2 \mathrm{~s}$. In the highest frequency band $(0.5-1 \mathrm{~Hz})$, the artifact is smaller and the beamforming images are roughly consistent with the MUSIC estimates, which explain the overall agreement of highfrequency back-projection studies.

At lower frequencies, the peak beamforming power migrates along the elongated ARF and is clearly dominated by the swimming effect. In contrast, in the MUSIC backprojection results, the dominant distribution of the seismic radiation in the down-dip region is clear in all frequency 

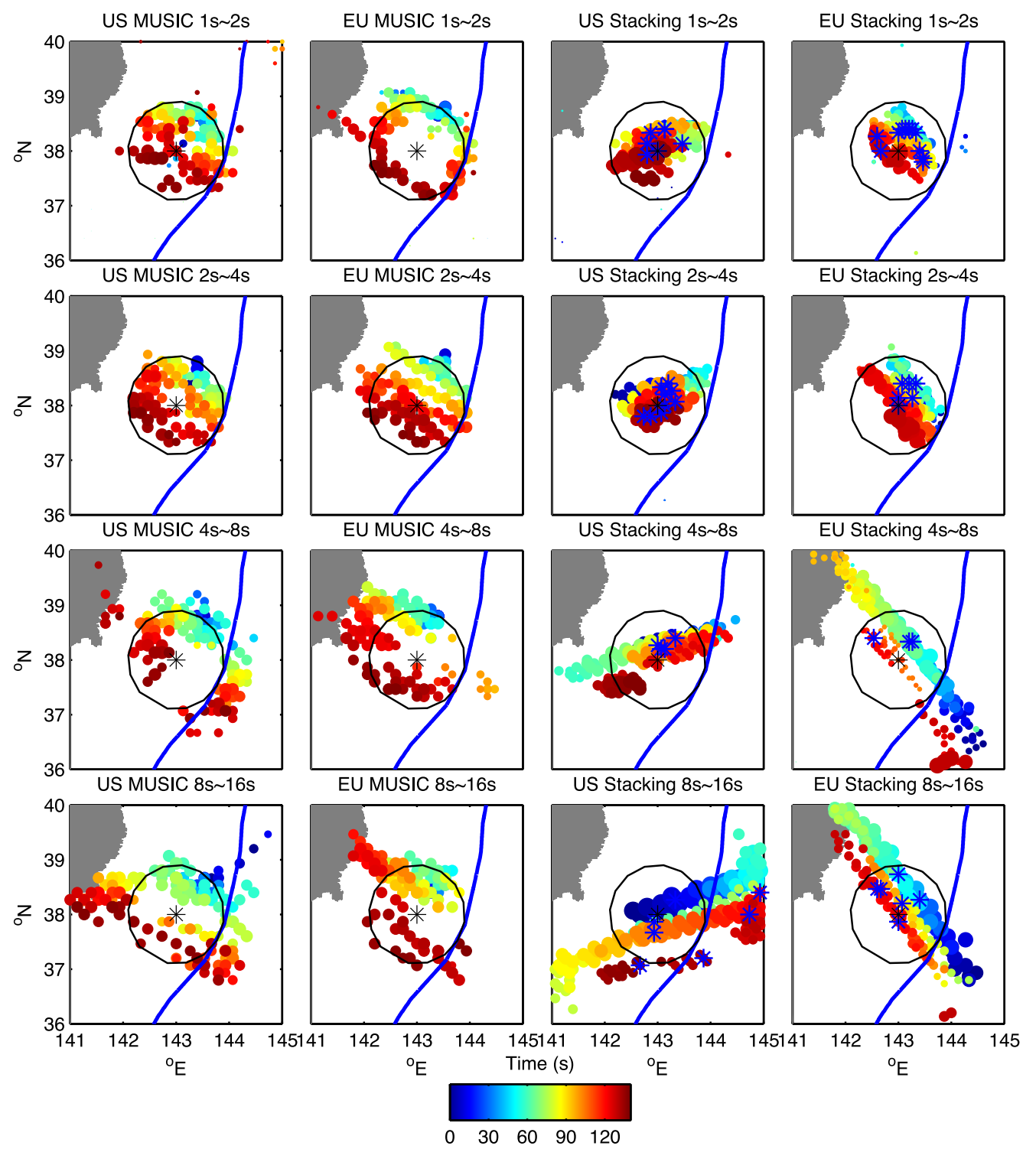

Fig. 5. Back-projection of circular rupture synthetics. The synthetic seismograms of a circular rupture scenario recorded at the USArray (left two
columns) and European array (right two columns) are processed by the MUSIC and linear stacking techniques separately at periods of $1 \sim 2 \mathrm{~s}, 2 \sim 4 \mathrm{~s}$, $4 \sim 8 \mathrm{~s}$ and $8 \sim 16 \mathrm{~s}$. The location of the aftershock is denoted by the black asterisk. The black circle is the hypothetical circular path of the rupture scenario. The solid circles are the peak of the back projection images color-coded by time and sized by their relative amplitude with respect to the global maximum. The blue asterisks are the "temporal local maximums" of the stacking result. The MUSIC recovers the rupture reasonably well at high frequency but loses the resolution beyond $4 \mathrm{~s}$. The time-domain stacking method shows a bias resulting from the swimming artifact.

bands at both arrays, except in the $8 \sim 16 \mathrm{~s}$ band which shows linear patterns corresponding to the artifact. We therefore restrict analysis of frequency-dependent source behavior based on MUSIC results to the band of $1 \sim 8 \mathrm{~s}$. As shown by the synthetic tests, even with the reference window strategy and the MUSIC method, the artifact at lower frequency is significant. For a given period, the radiators imaged by MUSIC with the European array are always to the west of those imaged with the USArray. At the USArray, an eastward migration of about $70 \mathrm{~km}$ is seen between 1 and $8 \mathrm{~s}$. This is consistent with the most clearly-reported frequency-dependent shifts by compressive sensing (Yao et al., 2011). However, this trend is absent in the European array results, which do not show a notable frequencydependent variation. This difference is consistent with the stronger artifact in the USArray, as shown in Fig. 5, due to the smaller signal to coda ratio resulting from its nodal orientation. Therefore, a frequency-dependent source location is not needed to explain the observations within the $1 \sim 8 \mathrm{~s}$ band. This indicates that any true frequency dependent source migration is minor compared to the $70 \mathrm{~km}$ shift apparent in the USArray back-projection.

\section{Summary and Discussion}

In this study, we explained the origin of the "swimming" artifact in array back-projection through the modified array response function and synthetic tests. We find that this effect is significant for time-domain stacking but can be efficiently mitigated by MUSIC array processing with a reference window strategy. MUSIC back-projection of the Tohoku-Oki earthquake recordings at the USArray and European array show no significant tendency for the source 

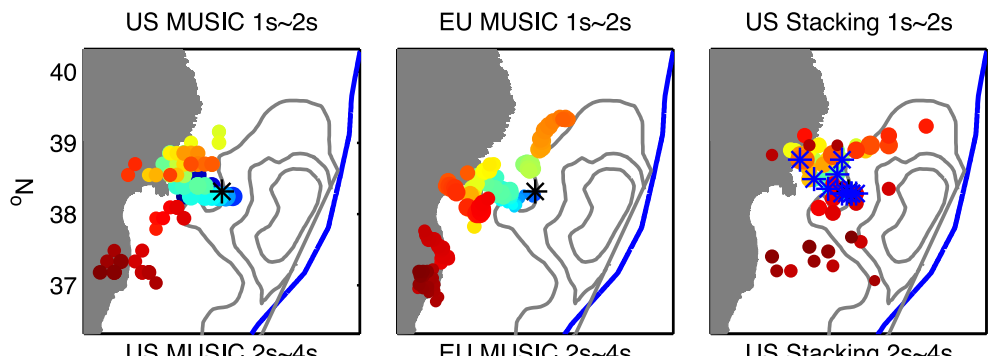

US Stacking 2s 4s
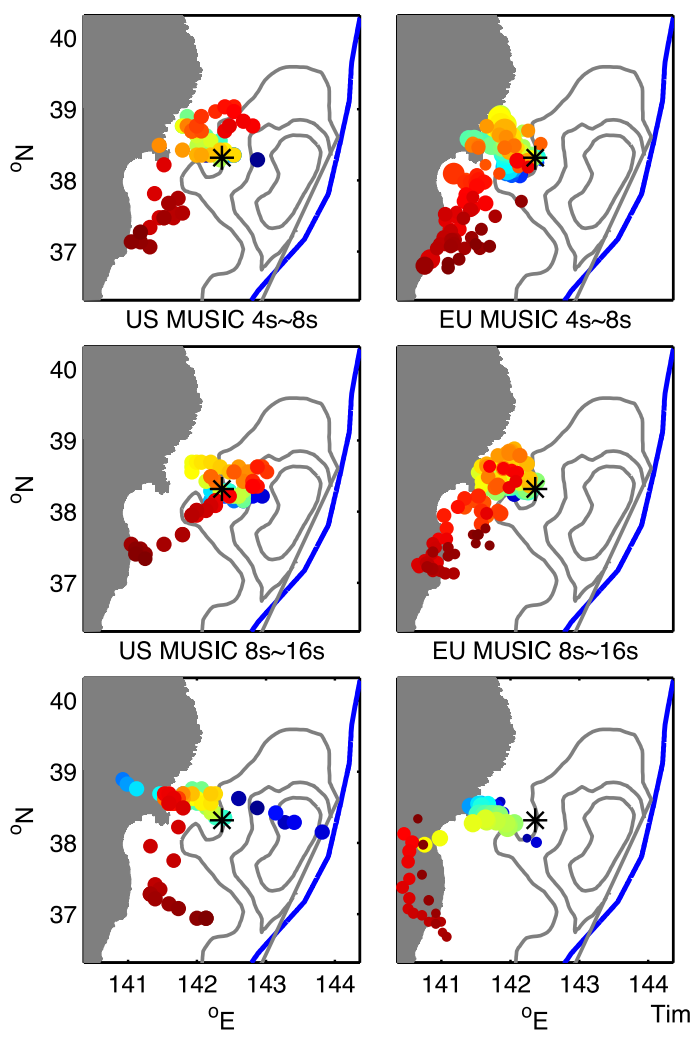
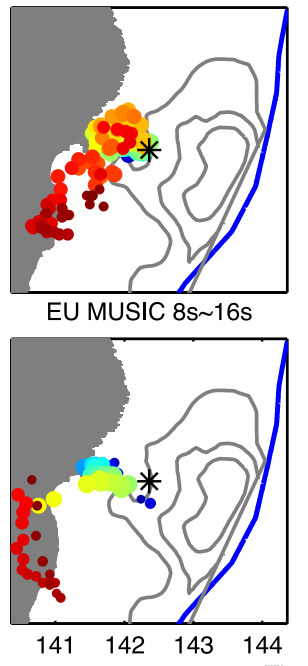

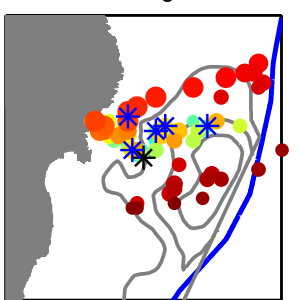

US Stacking $4 \mathrm{~s} \sim 8 \mathrm{~s}$

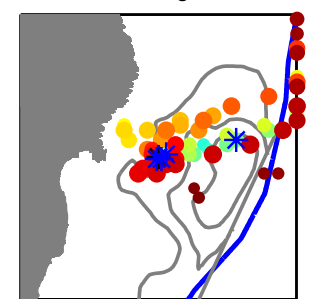

US Stacking $8 \mathrm{~s} \sim 16 \mathrm{~s}$

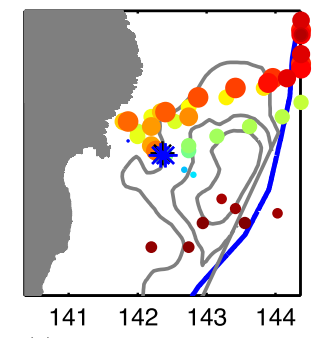

${ }^{\circ} \mathrm{E}$
EU Stacking 1s 2s

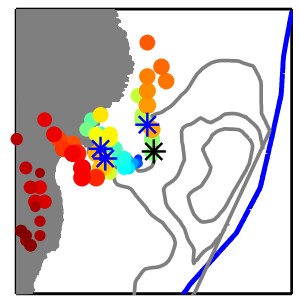

EU Stacking 2s 4s
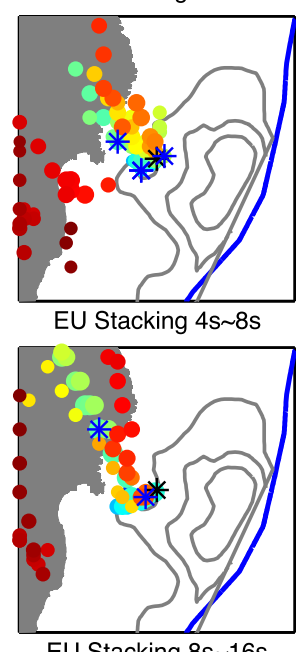

EU Stacking $8 s \sim 16 s$

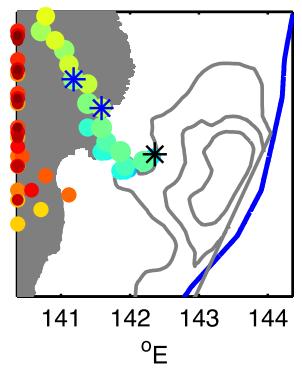

$0 \quad 30 \quad 60 \quad 90120150$

Fig. 6. Back-projection of the Tohoku-Oki earthquake at various periods ( $1 \mathrm{~s} \sim 16 \mathrm{~s})$ of the USArray and European array data with the linear stacking and MUSIC approaches. The hypocenter is denoted by the black asterisk. The location of the radiators are colored by time and sized by the amplitude with respect to the maximum. The blue asterisks are the "temporal local maxima" of the stacking result. The background grey lines are $12 \mathrm{~m}, 14 \mathrm{~m}$ and $36 \mathrm{~m}$ slip contours of a geodetic and seismic joint inversion model (Wei et al., 2012). The beamforming results are dominated by the artifact at periods longer than $4 \mathrm{~s}$. The MUSIC results are consistent over all frequency bands. Minor up-dip migration shows up in the period of $1 \mathrm{~s} \sim 8 \mathrm{~s}$ at the USArray, but is within the range expected from the "swimming" artifact and is absent in the European network.

to migrate towards the trench at increasing periods within the period band of $1 \sim 8 \mathrm{~s}$. An alternative interpretation is that the most substantial transition from deep to shallow slip occurs at periods longer than $16 \mathrm{~s}$, which implies an even longer rise time in the shallow portion of the megathrust. Furthermore, given that the swimming artifact is substantial in the time-domain stacking back-projections especially at long periods, and that this bias at the USArray behaves similarly to the potential frequency-dependent alongdip migration of slip, we consider that further tests and validations need to be performed before one can conclude that the along-dip slip migration can be observed by the low frequency back projection.

Our study also poses an interesting question: over which frequency band can we reliably image earthquake rupture processes by teleseismic back-projection? To image the
2011 Tohoku-Oki earthquake by the time-domain stacking technique with the USArray and European array, we determined that the frequency should be above $0.25 \mathrm{~Hz}$ to avoid substantial artifacts. As for the MUSIC technique, which suffers less severely from the swimming artifact, we suggest that the low-frequency results around $0.1 \mathrm{~Hz}$ can indicate first-order spatial patterns, but the finer details of the images are not reliable due to the limited resolution.

The reference window strategy needs to be compared with other efforts to mitigate the "swimming" effect. For instance, Koper et al. (2011b) proposed to reduce the strength of the artifact by selecting the temporal maxima among all the identified radiators. However, as we show in the synthetic circular rupture test, this approach cannot fully eliminate the frequency-dependent artifact when dealing with realistic rupture complexity involving, for instance, multiple 
simultaneous sources.

One mystery that remains to be explained is the discrepancy between the MUSIC and compressive sensing result (Yao et al., 2011). In principle, they are both performed in the frequency domain and share the reference window strategy, therefore both should suffer less from the swimming artifact. Yet, compressive sensing provide a large trenchward shift at $10 \mathrm{~s} \sim 20 \mathrm{~s}$, which is absent in the $8 \mathrm{~s} \sim 16 \mathrm{~s}$ band result with MUSIC. Efforts could be devoted to understand the other potential bias and artifact of various backprojection techniques. This concern also demonstrates the need to perform more rigorous synthetic tests involving more realistic rupture scenarios and crustal velocity models, which we will address in future work.

Acknowledgments. This research was supported by NSF grant EAR-1015704, by the Gordon and Betty Moore Foundation and by the Southern California Earthquake Center, which is funded by NSF Cooperative Agreement EAR-0106924 and USGS Cooperative Agreement 02HQAG0008. The EarthScope USArray and the European ORFEUS data center were used to access the data. This paper is Caltech Tectonics Observatory contribution \#198 and Caltech Seismolab contribution \#10075.

\section{References}

Asano, K. and T. Iwata, Strong Ground Motion Generation during the 2011 Tohoku-Oki Earthquake, AGU abstract, U42A-03, 2011.

Dunham, E. M., P. Favreau, and J. M. Carlson, A supershear transition mechanism for cracks, Science, 299(5612), 1557-1559, doi:10.1126/science.1080650, 2003.

Helmberger, D. V., Theory and application of synthetic seismograms, in Earthquakes: Observation, Theory and Interpretation, Soc. Ital. di Fis., Bolgna, Italy, 174-222, 1983.

Huang, Y., L. Meng, and J.-P. Ampuero, A dynamic model of the frequency-dependent rupture process of the 2011 Tohoku-Oki earthquake, Earth Planets Space, 64, this issue, 1061-1066, 2012.

Ishii, M., High-frequency rupture properties of the $M_{\mathrm{w}} 9.0$ off the Pacific coast of Tohoku Earthquake, Earth Planets Space, 63(7), 609-614, doi:10.5047/eps.2011.07.009, 2011.

Ishii, M., P. M. Shearer, H. Houston, and J. E. Vidale, Teleseismic P wave imaging of the 26 December 2004 Sumatra-Andaman and 28 March 2005 Sumatra earthquake ruptures using the Hi-net array, J. Geophys. Res.-Solid Earth, 112(B11), doi:10.1029/2006jb004700, 2007.

Kato, N., How frictional properties lead to either rupture-front focusing or cracklike behavior, Bull. Seismol. Soc. Am., 97, 2182-2189, 2007.

Koketsu, K. et al., A unified source model for the 2011 Tohoku earthquake, Earth Planet. Sci. Lett., 310(3-4), 480-487, doi:10.1016/j.epsl.2011.09.009, 2011.

Koper, K. D., A. R. Hutko, and T. Lay, Along-dip variation of teleseismic short-period radiation from the 11 March 2011 Tohoku earthquake (M(w) 9.0), Geophys. Res. Lett., 38, doi:10.1029/2011g1049689, 2011a.

Koper, K. D., A. R. Hutko, T. Lay, C. J. Ammon, and H. Kanamori, Frequency-dependent rupture process of the $2011 M_{\mathrm{w}} 9.0$ Tohoku Earthquake: comparison of short-period $P$ wave backprojection images and broadband seismic rupture models, Earth Planets Space, 63(7), 599-602, doi:10.5047/eps.2011.05.026, 2011, 2011 b.

Lay, T., H. Kanamori, C. J. Ammon, K. D. Koper, A. R. Hutko, L. Ye, H. Yue, and T. M. Rushing, Depth-varying rupture properties of subduction zone megathrust faults, J. Geophys. Res., 117, B04311, doi:10.1029/2011JB009133, 2012.

Lee, S. J., B. S. Huang, M. Ando, H. C. Chiu, and J. H. Wang, Evidence of large scale repeating slip during the 2011 Tohoku-Oki earthquake, Geophys. Res. Lett., 38, doi:10.1029/2011g1049580, 2011.

Meng, L. S., A. Inbal, and J. P. Ampuero, A window into the complexity of the dynamic rupture of the 2011 Mw 9 Tohoku-Oki earthquake, Geophys. Res. Lett., 38, L00g07, doi:10.1029/2011g1048118, 2011.

Miura, S., N. Takahashi, A. Nakanishi, T. Tsuru, S. Kodaira, and Y. Kaneda, Structural characteristics off Miyagi forearc region, the Japan Trench seismogenic zone, deduced from a wide-angle reflection and refraction study, Tectonophysics, 407, 165-188, 2005.

Miyake, H., Y. Yokota, H. Si, and K. Koketsu, Earthquake Scenarios Generating Extreme Ground Motions: Application to the 2011 Tohoku Earthquake, $A G U$ abstract, S 52B-07, 2011.

Mori, J., The Great 2011 Tohoku, Japan Earthquake (Mw9.0): An Unexpected Event, Southern California Earthquake Center Annual Meeting, Special Invited Talk, 2011.

Nakahara, H., Seismogram envelope inversion for high-frequency seismic energy radiation from moderate-to-large earthquakes, $A d v$. Geophys., 50, 401-426, doi:10.1016/S0065- 2687(08)00015-0, 2008.

Rost, S. and C. Thomas, Array seismology: Methods and applications, Rev. Geophys., 40(3), doi:10.1029/2000rg000100, 2002.

Roten, D., H. Miyake, and K. Koketsu, A Rayleigh wave back-projection method applied to the 2011 Tohoku earthquake, Geophys. Res. Lett., doi:10.1029/2011GL050183, 2011.

Sato, H. and M. C. Fehler, Seismic Wave Propagation and Scattering in the Heterogeneous Earth, Springer-Verlag and American Institute of Physics Press, 1998.

Sato, M., T. Ishikawa, N. Ujihara, S. Yoshida, M. Fujita, M. Mochizuki, and A. Asada, Displacement above the hypocenter of the 2011 Tohoku-Oki Earthquake, Science, 332(6036), 1395-1395, doi:10.1126/science.1207401, 2011.

Shao, G., X. Li, C. Ji, and T. Maeda, Focal mechanism and slip history of the $2011 \mathrm{Mw} 9.1$ off the Pacific coast of Tohoku Earthquake, constrained with teleseismic body and surface waves, Earth Planets Space, 63(7), 559-564, doi:10.5047/eps.2011.06.028, 2011.

Simons, M. et al., The 2011 magnitude 9.0 Tohoku-Oki Earthquake: Mosaicking the megathrust from seconds to centuries, Science, 332(6036), 1421-1425, doi:10.1126/Science.1206731, 2011.

Tromp, J., D. Komatitsch, and Q. Y. Liu, Spectral-element and adjoint methods in seismology, Commun. Comput. Phys., 3(1), 1-32, 2008.

Walker, K. T. and P. Shearer, Illuminating the near-sonic rupture velocities of the intracontinental Kokoxili Mw 7.8 and Denali Mw 7.9 strike-slip earthquakes with global P-wave back projection imaging, J. Geophys. Res., 114, doi:10.1029/2008JB005738, 2009.

Wang, D. and J. Mori, Rupture process of the 2011 off the Pacific coast of Tohoku Earthquake $\left(M_{\mathrm{w}} 9.0\right)$ as imaged with backprojection of teleseismic $P$-waves, Earth Planets Space, 63(7), 603607, doi:10.5047/eps.2011.05.029, 2011.

Wei, S., R. Graves, D. Helmberger, J.-P. Avouac, and J. L. Jiang, Sources of shaking and flooding during the Tohoku-Oki earthquake: A mixture of rupture styles, Earth Planet. Sci. Lett., doi:10.1016/j.eps1.2012.04.006, 2012.

Xu, Y., K. D. Koper, O. Sufri, and L. Zhu, Rupture imaging of the Mw 7.9 12 May 2008 Wenchuan earthquake from back projection of teleseismic P waves, Geochem. Geophys. Geosyst., 10, Q04006, Doi:10.1029/2008GC002335, 2009.

Yao, H. J., P. Gerstoft, P. M. Shearer, and C. Mecklenbrauker, Compressive sensing of the Tohoku-Oki Mw 9.0 earthquake: Frequency-dependent rupture modes, Geophys. Res. Lett., 38, doi:10.1029/2011gl049223, 2011.

Yao, H. J., P. Shearer, and P. Gerstoft, Subevent location and rupture imaging using iterative backprojection for the 2011 Tohoku Mw 9.0 earthquake, Geophys. J. Int., 2012 (submitted).

Yue, H. and T. Lay, Inversion of high-rate (1 sps) GPS data for rupture process of the 11 March 2011 Tohoku earthquake (M(w) 9.1), Geophys. Res. Lett., 38, doi:10.1029/2011g1048700, 2011.

Zerva, A. and V. Zervas, Spatial variation of seismic ground motions, Appl. Mech. Rev., 55(3), 271-297, 2002.

L. Meng (e-mail: 1smeng@gps.caltech.edu), J.-P. Ampuero, Y. Luo, W. $\mathrm{Wu}$, and $\mathrm{S}$. Ni 